\title{
Microsatellite variability in natural populations of the blackspot seabream Pagellus bogaraveo (Brünnick, 1768): a database to access parentage assignment in aquaculture
}

\author{
Andreia Lemos ${ }^{1}$, Ana Isabel Freitas ${ }^{2}$, Ana Teresa Fernandes ${ }^{2}$, Rita Gonçalves ${ }^{2}$, José Jesus ${ }^{1}$, Carlos \\ Andrade $^{3}$ \& António Brehm ${ }^{2}$ \\ ${ }^{1}$ Department of Biology, University of Madeira, Funchal, Portugal \\ ${ }^{2}$ Human Genetics Laboratory, University of Madeira, Funchal, Portugal \\ ${ }^{3}$ Centro de Maricultura da Calheta, Calheta, Portugal
}

Correspondence: A Brehm, Human Genetics Laboratory, University of Madeira, Campus of Penteada, 9000-390 Funchal, Portugal. E-mail: brehm@uma.pt

\begin{abstract}
Eight microsatellite loci previously reported were assessed for their utility in parentage assignment in 96 individuals belonging to natural populations of the blackspot seabream Pagellus bogaraveo (Brünnick, 1768) from the Mediterranean and Northeast Atlantic regions. At the mtDNA level, no differentiation was found between these two regions but based on microsatellite data an overall discrete genetic differentiation is perceivable between the two regions separated by the Strait of Gibraltar. The number of alleles per locus ranged from 8 to 30. A database constructed with allele frequency data from six populations was used in a simulation parentage assignment test using the software CERVUS. The test showed that the number of markers used is enough to perform parentage assignments with real data. The polymorphic information content for each locus was very high (mean value of 0.849), with a total exclusionary power of 0.9995. In summary, seven of the eight microsatellites analysed proved to be sufficient and powerful tools for parentage assignment in hatcheries and the allele frequency data given here can be used to perform pedigree analysis against which real data may be tested.
\end{abstract}

Keywords: Pagellus bogaraveo, microsatellites, genetic structure, parentage, database

\section{Introduction}

The worldwide exploitation of fish wild stocks has increased in the last decades to the extent that harvest- ing of natural populations is not now sufficient to supply the global demand for most species. Thus, the need for aquaculture production is in continuous demand, which implies the development of fish farming programmes and the establishment of successful breeding strategies. The sustainability of these strategies requires a precise knowledge of the genetic variability of wild populations from which individuals are selected to start breeding programmes (Knibb, Gorshkova \& Gorshkov 1998). Knowing individual genetic profiles is therefore essential if trait selection (e.g. growth rate, disease resistance, age at maturity) is an objective of such breeding programmes and also the only way a producer has to avoid efficiently, high rates of endogamy (inbreeding and reduced genetic variation), which will be important for long-term sustainable production (Olesen, Groen \& Gjerde 2000; Olesen, Gjedrem, Bentsen, Gjerde \& Rye 2003). Forensic identification of specimens usually requires a comparison of a known sample with a sample of unknown origin in order to quantify the probability of the unknown sample to have originated from the individual donating the known sample (Hammond, Jin, Zhong, Caskey \& Chakraborty 1994).

Much work has focused on the utility of molecular pedigree analysis in aquaculture (Norris, Bradley \& Cunningham 2000) or pedigree tracking in the selection of broodstock management procedures (Estoup, Gharbi, Sancristobal, Chevalet, Haffray \& Guyomard 1998; Evans, White \& Elliot 2000; Wilson \& Ferguson 2002). In those cases where it is not possible to 
exclude all but one parent (or both), the use of likelihood-based methods is necessary for parentage assignment, and simulation procedures must be performed to determine the significance of results (SanCristobal \& Chevalet 1997; Marshall, Slate, Kruuk \& Pemberton 1998; Gerber, Mariette, Streiff, Bodénès \& Kremer 2000). Likelihood-based methods consider all potential parents as possible candidates, avoiding the need to identify a known parent a priori. Microsatellites have been widely used to examine breeding systems in natural populations of several fish species (Olsen, Bentzen \& Seeb 1996; Fontaine \& Dodson 1999; Zane, Nelson, Jones \& Avise 1999; Dewoody, Fletcher, Wilkins \& Avise 2000; Gerber et al. 2000; Iyengar, Piyapattanakorn, Heipel, Stone, Howell, Child \& Maclean 2000; Norris et al. 2000). Microsatellites have also been used in parentage assignments (Hatchwell, Ross, Chaline, Fowlie \& Burke 2002) but always need to be first validated in wild animals and their polymorphic content estimated. These molecular markers are also useful to estimate levels of gene flow and thus population relatedness (Hansen, Kenchington \& Nielsen 2001), as well as to examine strain genetic structure (Liu, Chen \& Li 2005).

This paper focuses on the microsatellite genetic diversity of the blackspot sea bream Pagellus bogaraveo (Brünnick, 1768) from the Mediterranean Sea and the Atlantic Northeast region around the Azores and Madeira. The species is a protandrous hermaphrodite much in demand today for aquaculture breeding programmes. The possible role of the Strait of Gibraltar in promoting a genetic discontinuity of populations of several sparid species including P. bogaraveo was addressed by Bargelloni, Alarcon, Alvarez, Penzo, Magoulas, Reis and Patarnello (2003), who found a lack of variation within this species but not in other sparid species. Recently, a study focused on populations from the Azores Islands also found an absence of genetic diversity using mitochondrial markers and microsatellite analysis (Stockley, Menezes, Pinho \& Rogers 2005).

The objectives of the present study are: (1) to characterize eight microsatellite loci that have been previously described (Stockley, Rogers, Iyengar, Menezes, Santos \& Long 2000) for basic genetic parameters such as number of alleles and heterozygosity, (2) to assess whether these microsatellites show enough variability in $P$. bogaraveo to be useful in pedigree analysis or parentage assignment using forensic genetic indicators such as the polymorphism information content (PIC) and power of exclusion (PE), (3) to examine the distribution of variation among
$P$. bogaraveo populations based on microsatellite allele frequencies and (4) to present an allele frequency database to evaluate routine parentage testing data.

\section{Materials and methods}

\section{Sample collection}

Ninety-six individuals of $P$. bogaraveo were collected from four sites of the Atlantic Ocean (Ma-Madeira Island, $n=24$; Az-Azores Islands, $n=20$; NI-Northwest Iberian coast, $n=10$ and Al-Algarve/South of Portugal, $n=20$ ), and a Mediterranean sample $(\mathrm{Me}=22)$ composed of 12 specimens from Malta and 10 from the Ionian Sea.

\section{DNA extraction and amplification}

For DNA extraction, muscle samples from the dorsal region were collected and stored in $70 \%$ ethanol. In the case of populations from Madeira and Northwest Iberian Coast, blood was collected by punction and maintained at $-20{ }^{\circ} \mathrm{C}$. Tissue was homogenized in STE 100 buffer (0.1 M NaCl, 0.05 M Tris, 0.1 M EDTA; pH 8.0) and 10\% SDS. DNA was puried by extractions with phenol, phenol/chloroform (1:1), chloroform and precipitated with isopropanol (Sambrook, Fritsch \& Manitaris 1989). After cleaning with ethanol, DNA was suspended in water and kept at $-20{ }^{\circ} \mathrm{C}$. A partial mitochondrial cytochrome $b$ coding region was amplified in all individuals with universal primers from Kocher, Thomas, Meyer, Edwards, Paabo and Wilson (1989). The length of the coding-region polymerase chain reaction (PCR) products was approximately 370 base pairs (bp). Polymerase chain reaction fragments were sequenced in an ABI 310 sequencer; amplification and sequence protocols are available from the authors. Sequences were aligned using CLUSTAL x (Thompson, Higgins \& Gibson 1994) with minor adjustments made by eye. The sequences obtained were compared with two others available from GenBank (accession numbers AJ319818AJ276880).

Eight microsatellites (PbMS2, PbMS4, PbMS6, PBMS15, PbMS16, PbMS17, PbMS19 and PBS20) were amplified using the primers described by Stockley et al. (2000), which are based on a sample from the Azores. Polymerase chain reaction conditions were re-designed to adapt the annealing temperatures to our populations following difficulties in implementing those described by Stockley et al. (2000). Polymerase chain reaction products were separated by $6 \%$ 
polyacrylamide gel electrophoresis and visualized by silver staining. Fragment sizes were estimated in runs using DNAs with known sequences. Moreover, all individuals homozygous for a given allele at each locus were sequenced to determine the exact DNA sequence (both in structure as well as number of repeats) of the microsatellites. A DNA ladder made of an assortment of these homozygous fragments was constructed and used in subsequent polyacrilamide gel runs. The development of an allelic ladder with DNA fragments of the same size as the alleles found makes it possible to compare directly the runs using either manual or automated DNA electrophoresis. Microsatellite PbMS2 was not used for allele comparisons because it was found that although it had the same motif reported by Stockley et al. (2000), the sequence incorporates an insertion of 11 bp (TTATTCCCATC) not reported before, placed inside the repeat region immediately after the first CTT repeat. This insertion is widespread in all populations surveyed in fragments with eight to 16 repeats. Thus, amplified fragments with the same number of repeats appear with different lengths, depending on whether they incorporate the insertion or not, rendering its use impossible at a population scale. Microsatellite PbMS4 had a different repeat motif from that described by Stockley et al. (2000); three different motifs were found, each with variable number of repeats (Table 1). All sequenced alleles were submitted to GenBank and have accession numbers DQ520559-DQ520585.

\section{Statistical analyses}

Basic genetic parameters such as number of alleles at each microsatellite locus, percentage of polymorphic loci, proportion of individual heterozygous samples (direct count heterozygosity, $H_{0}$, as well as the unbiased estimate, $H_{\mathrm{e}}$ ) and population differentiation were calculated using GENEPOP V3.1D (Raymond \& Rousset 1995). The overall genetic diversity $\left(H_{\mathrm{T}}\right)$, within-population diversity $\left(H_{\mathrm{S}}\right)$ and between-population diversity $\left(D_{\mathrm{ST}}\right)$ were also calculated. Deviation from Hardy-Weinberg equilibrium per population and locus was calculated according to the Weir and Cockerham (1984) $F_{\text {IS }}$ estimator. The significance value of $F_{\text {IS }}$ was evaluated through a bootstrap technique based on random permutation of the original dataset, as implemented in FSTAT v.2.9.3. (Goudet 2001). This program uses a Bonferroni' correction for all significance levels (Rice 1989). The PIC and the PE for every locus were also calculated within each population and overall. PIC values describe how in-
Table 1 Characterization of eight Pagellus bogaraveo microsatellite loci based on 96 individuals

\begin{tabular}{|c|c|c|c|c|}
\hline Locus & $\begin{array}{l}\text { Homozygotes } \\
\text { sequenced }\end{array}$ & Repeat sequence & $\begin{array}{l}\text { Number } \\
\text { of } \\
\text { alleles }\end{array}$ & $\begin{array}{c}\text { Size } \\
\text { range } \\
(b p)\end{array}$ \\
\hline \multirow[t]{2}{*}{ PbMS2 } & 32 & (CTT)n & - & $*$ \\
\hline & 1 & $(\mathrm{CA}) \mathrm{nTA}(\mathrm{CA}) \mathrm{n}$ & & \\
\hline \multirow[t]{2}{*}{ PbMS4 } & 2 & $(\mathrm{CA}) n T(C A) n G G(C A) n$ & 16 & 162-194 \\
\hline & 2 & $(\mathrm{CA}) n T A(C A) n T A(C A) n$ & & \\
\hline PbMS6 & 2 & $(\mathrm{CA}) \mathrm{n}$ & 30 & $101-161$ \\
\hline PbMS15 & 2 & (CA)nGAG(TC)n & 24 & $157-203$ \\
\hline PbMS16 & 2 & $(\mathrm{CA}) \mathrm{n}$ & 22 & $147-193$ \\
\hline PbMS17 & 5 & $(G A) n$ & 8 & $187-201$ \\
\hline PbMS19 & 23 & $(C A G) n$ & 8 & $168-189$ \\
\hline PbMS20 & 10 & $(G T G C) n(G T) n$ & 20 & $148-188$ \\
\hline
\end{tabular}

* Size range varied among specimens due to the insertion of an $11 \mathrm{bp}$ segment (TTATTCCCATC) placed within the repeat region regardless of the number of repeats present.

formative a given genetic marker is and is described in Bolstein, White, Skolnik and Dawis (1980). The PE (or probability of exclusion) corresponds to the power with which a locus excludes an erroneously assigned individual from being the parent of an offspring (Weir 1996). Therefore, both PIC and PE are heterozygosity indicators. Two exclusion probabilities were calculated based on the allele frequency data: PE1 assumes genotypes are known for the offspring and a putative parent and, PE2 assumes that genotypes are known for the offspring, one confirmed parent and a putative parent. Both PE1 and PE2 are calculated according to Jamieson and Taylor (1997). An analysis of molecular variance (AMOVA) was performed based on Euclidean distances between haplotypes according to Excoffier, Smouse and Quattro (1992) using Arlequin (Schneider, Roessli \& Excoffier 2000). The total genetic variation was partitioned among two groups of individuals: Atlantic Ocean versus Mediterranean populations. Variance components, F-statistics and corresponding $P$-values were calculated by performing 10000 permutations of the original matrix data and comparing the results with the original values. A Mantel test available in the ARLEQUIN package (Schneider et al. 2000) was used to test for isolation by distance by providing a correlation coefficient of $F_{\text {ST }}$ and geographic distance matrices. Microsatellite frequency data from all individuals were merged to a single database, which was subsequently used to perform a simulation of parentage analysis for this set of loci using CERVus v 2.0 using the default parameters of the program (10 candidate parents, typing error $0.01 \%, 80 \%$ relaxed confidence and 95\% strict confi- 
dence, Marshall et al. 1998, Slate, Marshall \& Pemberton 2000). Basically, this software package simulates a parentage analysis estimating the resolving power of the set of loci and the critical values of the log-likelihood statistic $\Delta$ (Delta, LOD scores). An LOD score of zero implies that a putative father is as likely to be the true parent as any other randomly chosen one. The program allows for potential scoring errors that may occur in the dataset. These values can subsequently be used to test real data (descendants and parents), in a parentage analysis by evaluating the LOD scores of the most likely candidate parents against the likelihood of other unrelated candidate parents. CERVUS also allows the calculation of PE values according to two scenarios: PE1, in which genotypes are known for the offspring and a putative parent but genotypes are unavailable for the known parent (one parent missing), and PE2, in which genotypes are known for the offspring, one confirmed parent and one putative parent (both parents genotyped).

\section{Results and discussion}

\section{Cytochrome $b$ variability}

Amplification of the partial cytochrome $b$ gene fragment yielded a single haplotype that is similar to those existing in the Genbank. Given the fact that individuals belonged to Mediterranean and Atlantic locations, this result shows a remarkabley lack of variation in this mitochondrial region. This is surprising because all individuals sequenced were wild-caught pre-excluding the use of inbred specimens from aquaculture facilities. This is concordant with a previous survey by Bargelloni et al. (2003), which found only marginally significant differences between Atlantic and Mediterranean P. bogaraveo at the mtDNA level. Recently, Stockley et al. (2005) reported a lack of diversity in three populations from the north Atlantic region. Using D-Loop sequences, all haplotypes recovered were linked by a maximum of two mutational steps from the putative ancestral haplotype and the topology was a typical 'star-like' network indicative of a recently demographic expansion of a population, most probably from a small number of individuals (Fig. 2 from Slatkin \& Hudson 1991, Stockley et al. 2005).

\section{Microsatellite analysis}

All seven microsatellite loci were polymorphic in all the populations studied. Table 2 shows the genetic diversity $\left(H_{\mathrm{T}}\right)$ of $P$. bogaraveo and its distribution within
Table 2 Genetic diversity $\left(H_{\mathrm{T}}\right)$ of Pagellus bogaraveo and its distribution within $\left(H_{\mathrm{S}}\right)$ and between populations $\left(D_{\mathrm{ST}}\right)$

\begin{tabular}{|c|c|c|c|c|c|}
\hline \multirow[b]{2}{*}{ Locus } & \multicolumn{3}{|c|}{ Absolute values } & \multicolumn{2}{|c|}{ Relative values } \\
\hline & $H_{\mathrm{T}}$ & $H_{\mathrm{S}}$ & $D_{\mathrm{ST}}$ & $H_{\mathrm{T}}$ & $D_{\mathrm{ST}}$ \\
\hline PbMS4 & 0.894 & 0.886 & 0.008 & 0.896 & 0.010 \\
\hline PbMS6 & 0.958 & 0.960 & -0.002 & 0.957 & -0.003 \\
\hline PbMS15 & 0.939 & 0.943 & -0.004 & 0.938 & -0.005 \\
\hline PbMS16 & 0.940 & 0.938 & 0.003 & 0.941 & 0.003 \\
\hline PbMS17 & 0.707 & 0.714 & -0.007 & 0.705 & -0.009 \\
\hline PbMS19 & 0.721 & 0.667 & 0.054 & 0.732 & 0.065 \\
\hline PbMS20 & 0.924 & 0.900 & 0.024 & 0.929 & 0.029 \\
\hline Mean & 0.869 & 0.858 & 0.011 & 0.871 & 0.013 \\
\hline
\end{tabular}

$\left(H_{\mathrm{S}}\right)$ and between populations $\left(D_{\mathrm{ST}}\right)$ (Table 2$)$. A total of 128 different alleles were recovered over the seven loci for all populations (excluding PbMS2). The number of alleles per locus ranged from 8 to 30 (Table 1). Allele frequencies per locus in each population of $P$. bogaraveo and estimated genetic variability parameters are presented as supplementary material (www.uma.pt/bioarticles). All populations studied are at Hardy-Weinberg equilibrium and no genotypic disequilibrium was detected among pairs of loci. The observed genotypic frequencies do not differ statistically from the values expected under HW at each locus and for each population. The high genetic diversity explains in part the small $F_{\text {IS }}$ values that were either non-significant or changed sign among populations (Table 3). A small or negative value of $F_{\text {IS }}$ indicates that the population is not subjected to inbreeding and is not subdivided (Table 3). There was no correlation between geographical distances and $F_{\mathrm{ST}}$ values among populations (Mantel test, $r=0.37, P>0.05)$. Estimated genetic distances between populations were small (average $D_{\mathrm{ST}}=0.013$, Table 2). The Amova analysis confirms the lack of structure prevailing in $P$. bogaraveo populations. The clustering of populations according to their geographic locations yields an overall low but significant $F_{\text {ST }}$ value of $0.016(P<0.0001$, after 10000 permutations), indicating an insipient amount of population differentiation. The percentage of variation attributed to differences among populations within groups was only $1.2 \%$; $0.49 \%$ was explained as variation among groups and $98.3 \%$ was attributed to differences within populations. This shows that the populations from the Mediterranean and Atlantic regions do not differ significantly and most of the variability found is due to within-population variation. Also, the high within-population variation is most probably the explanation for the high gene diversity values found $\left(H_{\mathrm{o}}\right)$. The 
Table 3 Within-population genetic variation of six Pagellus bogaraveo populations based on seven loci

\begin{tabular}{|c|c|c|c|c|c|c|c|}
\hline Population & MNA & $H_{\mathrm{o}}$ & $H_{\mathrm{e}}$ & $F_{\text {IS }}$ & PIC & PE1 & PE2 \\
\hline $\mathrm{Ma}$ & 13.57 & 0.816 & 0.873 & 0.067 & 0.839 & 0.9991 & 0.9999 \\
\hline$A z$ & 10.86 & 0.814 & 0.835 & 0.026 & 0.788 & 0.9967 & 0.9998 \\
\hline $\mathrm{NI}$ & 9.71 & 0.886 & 0.862 & -0.030 & 0.796 & 0.9966 & 0.9998 \\
\hline $\mathrm{Me}$ & 13.14 & 0.838 & 0.866 & 0.033 & 0.828 & 0.9987 & 0.9999 \\
\hline $\mathrm{Al}$ & 11.86 & 0.864 & 0.864 & 0.000 & 0.826 & 0.9985 & 0.9999 \\
\hline Mean & & & & $\begin{array}{r}0.020 \\
( \pm 0.013)\end{array}$ & 0.849 & 0.9995 & 0.9998 \\
\hline
\end{tabular}

MNA, mean number of alleles; $H_{\mathrm{o}}$ and $H_{\mathrm{e}}$ are the population's observed and expected heterozigosity. $F_{\text {IS }}$ values follow the formulae of Weir and Cockerham (1984); PIC is the mean value over all loci. PE1 and PE2 are the total exclusion power of the first parent and the second parent respectively.

highest value of $F_{\mathrm{ST}}$ was recorded between the Malta and the Azorean populations $\left(F_{\mathrm{ST}}=0.032\right.$, $P<0.0001)$ and the lower were for the pairs Madeira/Algarve, Northwest Iberian/Azores and Ionian Sea/Azores, with non-significant $P$ values. In the simulation of the parentage analysis, we used the default values of CERVus (10 000 replicates or simulated offspring, 10 candidate males of being the true parent, all loci typed and a typing error of 0.01). Given both scenarios of PE1 and PE2, our database gives an average LOD score in the first scenario of 4.41 higher than the LOD score of the next most likely male $99.6 \%$ of the time ( $\mathrm{SD}=1.52)$. In the second scenario, this value is $1.96(\mathrm{SD}=1.01) 95.12 \%$ of the time. For each of the seven loci, the PIC value was high enough for this set of loci to be considered powerful for determining parentage when using real data (PIC mean value was 0.849 ) with a total exclusionary power (PE) of 0.9995. Moreover, the values of PE1 and PE2 are all above 99\%, which indicates that the individuals analysed are part of one single population (corroborating mtDNA information) and that the number of specimens analysed was enough to recover most of the allelic variation of the loci analysed.

\section{Conclusion}

The present study analyses microsatellite diversity at seven loci in six Atlantic and Mediterranean populations of $P$. bogaraveo. The populations show high levels of genetic diversity albeit low genetic differentiations, suggesting that these populations are not isolated. This core set of seven microsatellite allele frequencies represents a powerful and efficient method to use in any pedigree analysis for local hatcheries to quantify the number and genetic profile of founders and can therefore be applied for both parentage assignment and trait selection programmes.

\section{Acknowledgments}

The authors are grateful to Dr Jon Slate for comments on CERvus and to all those who provided us with fish specimens, and to one anonymous referee for must useful comments in an earlier version of the manuscript. This study was partially financed by project MARINOVA, contract MAC/4.2/M11 (INTERREG).

\section{References}

Bargelloni L., Alarcon J., Alvarez M., Penzo E., Magoulas A., Reis C. \& Patarnello T. (2003) Discord in the family Sparidae (Teleostei): divergent phylogeographical patterns across the Atlantic-Mediterranean divide. Journal of Molcular Evolution 16, 1149-1158.

Bolstein D., White R., Skolnik M. \& Dawis R. (1980) Construction of a genetic linkage map using restriction fragment length polymorphisms. American Journal of Human Genetics 32, 314-331.

DeWoody J., Fletcher E., Wilkins D. \& Avise J. (2000) Parentage and nest guarding in the tessellated darter (Etheostoma olmstedi) assayed by microsatellite markers (Perciformes: Percidae). Copeia 3, 740-747.

Estoup A., Gharbi K., SanCristobal M., Chevalet C., Haffray P. \& Guyomard R. (1998) Parentage assignment using microsatellites in turbot (Scophthalmus maximus) and rainbow trout (Oncorhynchus mykiss) hatchery populations. Candian Journal of Fisheries and Aquatic Sciences $\mathbf{5 5}$, 715-725.

Evans B.,White R. \& Elliot N. (2000) The use of microsatellite markers for parentage analysis in Australian blacklip and hybrid abalone. Journal of Shellfish Research 19, 511.

Excoffier L., Smouse P.E. \& Quattro J.M. (1992) Analysis of molecular variance inferred from metric distances among DNA haplotypes: application to human mitochondrial DNA restriction data. Genetics 131, 479-491.

Fontaine P. \& Dodson J. (1999) An analysis of the distribution of juvenile Atlantic salmon (Salmo salar) in nature as a function of relatedness using microsatellites. Molecular Ecology 8, 189-198. 
Gerber S., Mariette S., Streiff R., Bodénès C. \& Kremer A. (2000) Comparison of microsatellites and amplified fragment lenght polymorphism markers for parentage analysis. Molecular Ecology 9, 1037-1048.

Goudet J. (2001) FSTAT v.2.9.3.: a computer to estimate and test gene diversities and fixation indeces. Available from $\langle$ http://www.unil.ch/izea/softwares/fstat.html $\rangle$.

Hammond A., Jin L., Zhong Y., Caskey T. \& Chakraborty R. (1994) Evaluation of 13 short tandem repeat loci for use in personal identification applications. American Journal of Human Genetics 55, 175-189.

Hansen M., Kenchington E. \& Nielsen E. (2001) Assigning individual fish to populations using microsatellite DNA markers. Fish and Fisheries, Series 2, 93-112.

Hatchwell B., Ross D., Chaline N., Fowlie M. \& BurkeT. (2002) Parentage in the cooperative breeding system of long-tailed tits, Aegithalos caudatus. Animal Behaviour 64, 55-63.

Iyengar A., Piyapattanakorn S., Heipel D., Stone D., Howell B., Child A. \& Maclean N. (2000) A suite of highly polymorphic microsatellite markers in turbot (Scophthalmus maximus L.) With potential for use across several flatfish species. Molecular Ecology 9, 368-371.

Jamieson A. \& Taylor St. C.S. (1997) Comparison of three probability formulae for parentage exclusion. Animal Genetics 28, 397-400.

Knibb W., Gorshkova G. \& Gorshkov S. (1998) Selection and crossbreeding in Mediterranean cultured marine fish. pp 47-60 In: Proc. TECAM Seminar on Genetics and Breeding of Mediterranean Aquacultured Species. (ed. by D.M. Bartley \& B. Basurco). Zaragoza, Spain.

Kocher T.,Thomas W., Meyer A., Edwards S., Paabo S. \& Wilson A. (1989) Dynamics of mitochondrial DNA evolution in animals: amplification and sequencing with conserved primers. Proceedings of National Academic of Science USA 86, 6169-6200

Liu Y., Chen S. \& Li B. (2005) Assessing the genetic struture of three Japanese flounder (Paralichthys olivaceus) stocks by microsatellite markers. Aquaculture 243, 103-111.

Marshall T., Slate J., Kruuk E. \& Pemberton J. (1998) Statistical confidence for likelihood-based paternity inference in natural populations. Molecular Ecology 7, 639-655.

Norris A., Bradley D. \& Cunningham E. (2000) Parentage and relatedness determination in farmed Atlantic salmon (Salmo salar) using microsatellite markers. Aquaculture 182, 73-83.

Olsen J., Bentzen P. \& Seeb J. (1996) Characterization of seven microsatellite loci derived from pink salmon. Molecular Ecology 7, 1087-1089.

Olesen I., Groen A. \& Gjerde B. (2000) Definition of animal breeding goals for sustainable production systems. Journal of Animal Science 78, 570-582.
Olesen I., Gjedrem T., Bentsen H., Gjerde B. \& Rye M. (2003) Breeding programs for sustainable aquaculture. Journal of Applied Aquaculture 13, 179-204.

Raymond M. \& Rousset F. (1995) An exact test for population differentiation. Evolution 49, 1280-1283.

Rice R. (1989) Analysing tables of statistical tests. Evolution 43, 223-225.

Sambrook J., Fritsch E. \& Manitaris T. (1989) Molecular Cloning: A Laboratory Manual, 2nd edn. Cold Spring Harbor Laboratory Press, Plainview, NY, USA.

SanCristobal M. \& Chevalet C. (1997) Error tolerant parent identification from a finite set of individuals. Genetic Research 70, 53-62.

Schneider S., Roessli D. \& Excoffier L. (2000) Arlequin, ver. 2.000: A Software for Population Genetics Data Analysis. Genetics and Biometry Laboratory, University of Geneva, Switzerland.

Slate J., Marshall T. \& Pemberton J. (2000) A retrospective assessment of the accuracy of the paternity inference program CERVUS. Molecular Ecology 9, 801-808.

Slatkin M. \& Hudson R. (1991) Pairwise comparisons of mitochondrial sequences in stable and exponentially growing populations. Genetics 129, 555-562.

Stockley B., Rogers A., Iyengar A., Menezes G., Santos R. \& Long A. (2000) Ten microsatellite loci isolated and developed for the blackspot seabream Pagellus bogaraveo (Brünnich, 1768). Molecular Ecology 9, 993-1011.

Stockley B., Menezes G., Pinho R. \& Rogers A. (2005) Genetic population structure in the black-spot sea bream (Pagellus bogaraveo Brünnich, 1768) from the NE Atlantic. Marine Biology 146, 793-804.

Thompson J., Higgins D. \& Gibson T. (1994) Clustal W: improving the sensitivity of progressive multiple sequence alignment through sequence weighting, position specific gap penalties and weight matrix choice. Nuclear Acid Research 22, 4673-4680.

Weir B. (1996) Genetic Data Analysis II. Methods for Discrete Population Genetic Data. Sinauer Associates, Sunderland, MA., USA.

Weir B. \& Cockerham C. (1984) Estimating F-statistics for the analysis of population structure. Evolution 38, 1358-1370.

Wilson A. \& Ferguson M. (2002) Molecular pedigree analysis in natural populations of fishes: approaches, applications, and practical considerations. Canadian Journal Fisheries and Aquatic Sciences 59, 1696-1707.

Zane L., Nelson W., Jones A. \& Avise J. (1999) Microsatellite assessment of multiple paternity in natural populations of a live-bearing fish, Gambusia holbrooki. Journal of Evolution Biology 12, 61-69. 